\title{
eJRIEPS
}

Ejournal de la recherche sur l'intervention en éducation physique et sport

Hors-série $\mathrm{N}^{\circ} 2 \mid 2018$

À propos de certaines bases théoriques et pratiques des sports collectifs

\section{Initiative et expérience au cœur de la pensée tactique en sport collectif}

Jean-Francis Gréhaigne

\section{(e) OpenEdition \\ 1 Journals}

Édition électronique

URL : http://journals.openedition.org/ejrieps/510

DOI : 10.4000 /ejrieps. 510

ISSN : 2105-0821

Éditeur

ELLIADD

Référence électronique

Jean-Francis Gréhaigne, «Initiative et expérience au cœur de la pensée tactique en sport collectif » eJRIEPS [En ligne], Hors-série № 2 | 2018, mis en ligne le 01 juillet 2018, consulté le 01 août 2019. URL : http://journals.openedition.org/ejrieps/510 ; DOI : 10.4000/ejrieps.510

La revue eJRIEPS est mise à disposition selon les termes de la Creative Commons Attribution 4.0 International License. 


\section{Initiative et expérience au cœur de la pensée tactique en sport collectif}

Dans les sports collectifs, les règles premières régissent les conditions de déroulement d'un jeu jusqu'à la victoire et fondent la logique du jeu dans la prise en compte systématique du rapport d'opposition entre une attaque et une défense. Les conséquences de cette conception du jeu sont considérables. Les multiples possibilités de placement, de déplacements et d'interactions donnent au jeu un caractère d'incertitude, renforcé par le fait que l'initiative change de camp avec le gain ou la perte de la balle (Gréhaigne \& Dietsch, 2015). Pour Crozier et Friedberg (1977), ce véritable «système d'actions concret» est un ensemble humain structuré qui coordonne les actions de ses participants par des mécanismes d'interactions organisées de façon relativement stables mais bien sûr évolutives dans le temps. Comprendre comment les joueurs agissent dans ce système d'action, fait de rapports de forces et de relations d'opposition, constitue un passage obligé pour tous les chercheurs qui investiguent la singularité des sports collectifs. On vise ici des fonctions de compréhension pour répondre de façon cohérente et appropriée aux problèmes qui se posent en jeu. Dans les discours sur le jeu, on parle souvent d'initiatives et d'intentions sans bien différencier ces deux termes et, souvent, l'expérience du joueur ainsi que la pensée tactique sont convoquées pour justifier l'emploi de ces concepts.

Dans ce texte, nous allons revenir sur l'utilisation de ces différents concepts dans les sports collectifs afin de mieux en comprendre leur utilité et leur emploi. En effet, l'importance des processus cognitifs conscients ou juste infra-conscients pour analyser les rapports d'opposition n'est plus à démontrer (Deleplace, 1979 ; Gréhaigne \& Godbout, 1995). Chez le joueur, adopter ce mode de fonctionnement de la pensée suppose qu'il est attentif aux processus et aux dynamismes en évolution dans le rapport de forces momentané afin que ses initiatives, voire ses intentions, soient couronnées de succès.

\section{Intention et initiative}

Une intention peut être définie comme l'envie ou la résolution prise par un joueur ou une joueuse d'agir pour d'atteindre un ou plusieurs objectifs fixés à l'avance, sa décision de passer à l'action étant quasiment définitive. Menaut (1998) parle d'une vision anticipée car «les projets ou les intentions concernent l'élaboration d'un plan en devenir dont les objectifs sont clairement définis et articulés aux moyens pour y parvenir » (p. 321). C'est donc une disposition d'esprit par laquelle on se propose délibérément un objectif. Mais on peut très bien en rester à une activité motrice limitée aux prémices moteurs d'une séquence comportementale qui n'ira pas plus loin. Dans le jeu, avoir des intentions ne suffit pas car les joueurs ne peuvent pas en rester longtemps au niveau du projet, des 
rêves ou des illusions irréalistes. Ils doivent se confronter aux réalités du rapport d'opposition.

Une initiative consiste en l'action d'un joueur qui fait quelque chose ou c'est la qualité de quelqu'un qui entreprend les choses, prend des décisions par soi-même ou pour ses équipiers, sans recourir à l'avis, au conseil de quelqu'un d'autre. Le joueur sait prendre rapidement la décision nécessaire. En effet, chaque jeu sportif consiste en une lutte permanente entre l'attaque et la défense. L'avantage de l'attaque est fondé sur la possession du ballon, condition sine qua non pour la réalisation d'un point. Ici, les initiatives du porteur de balle sont particulièrement importantes. Par contre, la défense cherche à contrecarrer les initiatives des attaquants tout en cherchant à récupérer le ballon. L'application de ce principe suppose du dynamisme, de l'agressivité et aussi de l'initiative.

Pour le joueur, l'intention est dans le devenir, l'initiative est dans le champ d'action immédiat. Au plan du temps et de la décision, c'est donc assez différent. Avec l'intention, la conscience formule une demande à propos de l'action qu'elle a choisie d'investiguer mais le système de résolution de la tâche répond à l'intention selon les possibilités du moment, donc pas toujours dans le sens désiré. Ces éléments nous conduisent à considérer nécessaire de privilégier l'initiative chez les joueurs car porteuse de progrès versus une intention qui peut relever de la chimère. Cela représente la base même de l'efficacité en sport collectif que de permettre, pour tous les participants d'une même équipe, le plein déploiement de l'initiative individuelle. Ici, il n'y a pas opposition entre initiative individuelle et actions collectives si la possibilité d'initiative existe pour tous les joueurs et à chaque instant.

Pour Deleplace (1979), avec un «système représentatif mental commun des informations (sur le jeu), non seulement leur ensemble complexe devient significatif, interprétable pour chacun, mais du même coup l'initiative de chacun devient elle-même facilement interprétable, compréhensible, significative pour chacun des autres partenaires, et cela à tout moment » (p. 18). Cette initiative qui, à la vitesse des idées, ne présente qu'un contenu fugitif, s'inscrit toutefois dans un acte qui se coordonne ou va se coordonner sur le terrain. Or, une initiative n'est pas isolée. Elle prend le temps de naître et de se développer dans d'autres actions. Elle peut être également une réponse à celles des adversaires ou des partenaires et cette initiative se développe dans le temps en fonction de projets variés voire contradictoires (Fernandez, 2002). Il s'agit de considérer une multiplicité d'évolutions permettant de penser les jeux sportifs collectifs d'opposition à partir des échanges entre sousgroupes et équipes. Bien sûr, ces processus sont en boucle entre plusieurs niveaux de décision et d'initiatives dont l'emboîtement temporel rend compte du cheminement de la pensée et des actions du ou des joueurs impliqués.

Passer d'avoir des intentions à prendre des initiatives, c'est passer de la stratégie à la tactique, c'est quitter le domaine de la réflexion pour aller vers l'action concrète. En sport collectif, la terminologie considère que la stratégie représente les éléments discutés à l'avance pour s'organiser 
en fonction des caractéristiques propres de l'équipe adverse et de ses individualités. Selon, Gréhaigne, Godbout, \& Bouthier (1999) «la stratégie peut soit concerner les grandes options de jeu, soit spécifier l'intervention des joueurs pour différentes classes de situations de jeu» (p. 160). Il s'agit d'une anticipation liée aux informations dont on dispose sur les adversaires. Par ailleurs, la tactique dans le jeu se construit au cours de l'action en modifiant, selon les enseignements des événements, la perception d'informations ou la conduite envisagée. La tactique suppose la mobilisation chez le sujet des capacités à utiliser pour l'action au regard de multiples déterminants et des aléas extérieurs. On peut la définir (Gréhaigne, 2014) comme la forme d'actions propre au sujet en situation de jeu où, pour remplir son rôle, le joueur s'efforce de subir au minimum les contraintes, les incertitudes et les hasards du jeu et d'utiliser au maximum les capacités, potentiels et compétences dont il dispose. L'initiative constitue alors une composante décisive en sport collectif car elle permet d'imposer ses choix et non de subir des relations d'opposition voulues par l'adversaire.

L'approche que nous proposons dans ces lignes s'inspire ainsi des théories de la complexité en relation avec l'apport de la réflexion sur les systèmes dynamiques dans les jeux (Gréhaigne \& Godbout, 2014). Toutefois, cette posture épistémologique qui vise à appréhender la complexité, constitue selon nous l'originalité, l'intérêt, mais aussi la difficulté de la démarche. «La pensée complexe est donc essentiellement la pensée qui traite avec l'incertitude, et qui est capable de concevoir l'organisation. C'est la pensée capable de relier, de contextualiser, de globaliser, mais en même temps de reconnaître le singulier, l'individuel, le concret » (Le Moigne, 1999, p. 258). La possibilité de progrès repose précisément sur la reconnaissance du rôle déterminant, parce que premier chez l'être humain, de la pensée organisatrice abstraite de l'action, c'est-à-dire, dans le jeu, de la pensée tactique.

\section{L'intuition, le flair}

Pour Bourdieu (1980), le «sens pratique »n'est pas autre chose que la logique pratique à la fois commode et tournée vers l'action. L'auteur nous propose une théorie de l'action qui n'est pas une fin en elle-même, mais qui est la condition d'une interprétation adéquate des pratiques. Ainsi, Bourdieu compare le sens pratique au «sens du jeu en sport» pour un joueur qui agit, par moment, sans recul réflexif, ni calcul stratégique vraiment conscient. Cette maîtrise pratique de la logique du jeu ne s'acquiert que par l'expérience, et fonctionne parfois en deçà de la conscience et du discours (Mouchet, 2003). Néanmoins, soulignons que l'arrière-plan cognitif intervient fréquemment dans la recherche des indices nécessaires au traitement d'une configuration du jeu, avec les situations analogues déjà vécues comme référence principale. L’intuition est une « vérité » qui se présente à la pensée avec la clarté d'une évidence, qui servira de fondement à un raisonnement ultérieur. 
Au sens figuré, le flair désigne une forme de perspicacité, qui consiste en la capacité apparemment intuitive à épouser les circonstances pour tirer parti de ces situations. En rugby, le «french-flair » consistait en cette capacité à déclencher une action improbable à tout moment, se concrétisant par des « essais venus de nulle part» (Bouthier, Barthes, David, \& Gréhaigne, 1994). Au centre de la connaissance, il y a le joueur connaissant qui est, en même temps, un observateur, un concepteur et un acteur. Cela recouvre chez le joueur une forme d'intelligence tactique (Gréhaigne \& Godbout, 2014) qui permet :

- de simplifier tout en respectant la complexité du jeu ;

- d'analyser l'évolution des configurations du jeu à partir d'indices partiels ;

- de hiérarchiser l'important, le significatif de l'accessoire ;

- de flairer les bons coups et de se servir du hasard de façon favorable ;

- de solliciter la mémoire, l'imagination et l'expérience du joueur.

Les actions en jeu relèvent principalement de l'intelligence pratique voire de la ruse, où les gestes et les décisions se révèlent au gré des situations et se programment peu. L'expression «l'œil du maquignon », habituellement attribuée aux entraîneurs chevronnés, peut très bien représenter une qualité chez l'ensemble des protagonistes. Cela souligne bien que si l'œil avisé est si important pour le joueur, alors il faut accorder un intérêt particulier à cette forme d'intelligence qui vise, avant tout, l'efficacité pratique. Celle-ci s'applique à des réalités momentanées et changeantes qui ne se prêtent guère à la mesure précise ou à un raisonnement détaillé demandant du temps. Or, toutes les compétences dont est faite l'intelligence pratique sont le plus souvent ramenées au niveau des qualités personnelles et psychologiques du joueur ou de routines acquises avec le temps. Nous sommes par conséquent dans l'ingéniosité du geste qui se construit dans l'instant. Aussi, ces « raccourcis fulgurants» sont des données qui permettent aux experts de trouver une tactique adéquate sans inventorier et comparer toutes les stratégies possibles. C'est en ce sens une forme d'intelligence et de pensée qui implique un ensemble complexe et très cohérent de connaissances, d'attitudes mentales, de comportements intellectuels et de compétences motrices qui combinent le flair, la sagacité, la feinte, la débrouillardise, l'attention vigilante et le sens de l'opportunité grâce une expérience longue à acquérir. Ces fonctionnements heuristiques permettent de maintenir l'efficacité dans des situations caractérisées par une masse importante de données à prendre en compte. Un fonctionnement basé sur une «logique floue» suppose des compétences qui transcendent les connaissances, savantes ou non, déclaratives ou procédurales en relation avec une bonne expérience.

\section{L'expérience}

Au cours du temps, l'expérience du joueur devient un élément important de la qualité de la pensée 
tactique, et l'expérience peut aider à construire la capacité de prendre de meilleures décisions en améliorant la prise de décision par l'accumulation des réponses qui ont entraîné des réussites. Dans la langue française, le mot expérience a deux significations distinctes. Tout d'abord, expérience, au singulier, signifie d'une manière générale et abstraite, l'instruction acquise par le fait de vivre et d'enregistrer des événements, des phénomènes. Quand on applique à un enseignant le mot expérience pris au singulier, il exprime l'instruction qu'il a acquise par l'exercice de son métier. C'est dans ce sens qu'on dit qu'un homme a acquis de l'expérience, qu'il a de « l'expérience ». Le résultat de cette acquisition est un ensemble des connaissances concrètes acquises par l'usage et le contact avec la réalité de la vie, et prêtes à être mises en œuvre. Ensuite, par extension, on a donné dans un sens concret le nom «d'expérience» aux faits qui nous fournissent cette instruction expérimentale des choses.

On différencie donc l'expérience volontaire qui fait l'objet d'une attention spécifique, d'une volonté d'accumuler des connaissances, de l'expérience courante qui relève du fait que l'on vit et qui s'accumule sans intention spécifique d'apprendre et qui fonctionne en tâche de fond. Ainsi, la source première de notre connaissance est l'expérience. Pour qu'il y ait expérience, il faut que nous ayons perçu une chose elle-même. Mais doit-on distinguer perception et expérience ? Pour répondre à cette question, on doit envisager la perception comme celle d'un objet unique. L'expérience au contraire est une perception répétée et volontaire. La raison édicte des règles et organise et structure l'expérience en matière de connaissances car l'expérience lui est subordonnée.

Avec Mouchet (2003), nous noterons que le concept d'expérience est très proche de celui d'habitus (Bourdieu, 1980), tout en sous-tendant une dimension plus personnelle et un rôle actif du sujet dans son élaboration. Une «sphère » désigne l'ensemble des personnes avec lesquelles un individu donné entretient des relations, qu'elles soient publiques, ou privées. L'individu y est bien au centre, mais le type de relations et les règles qui les régissent permettent d'établir des distinctions.

- La sphère publique ou socio-culturelle de l'expérience est en rapport avec le groupe social considéré et pour ce qui nous concerne le groupe de joueurs. Nous pouvons considérer que le partage de l'expérience est constitué par un ensemble d'éléments tels que les normes et valeurs, les croyances, les attitudes, les savoirs, le référentiel commun d'analyse. Ces éléments plus ou moins communs permettent aux joueurs d'un même groupe de vivre une même situation, avec une interprétation en partie identique des configurations du jeu. Alors se pose immédiatement la question de la construction d'une expérience collective qui dépasse, bien sûr, les juxtapositions des expériences individuelles.

- La sphère privée constitue l'autre pôle de l'analyse : l'idée de sphère privée est d'abord pensée comme le domaine du privatif, avec peu d'interactions avec autrui pour protéger sa vie privée. Dans ce cas, la vie privée est la capacité, pour une personne ou pour un groupe de 
personnes, de s'isoler afin de protéger ses intérêts. Les limites de la sphère privée ainsi que ce qui est considéré comme privé diffèrent selon les groupes, les cultures, les individus ainsi que selon les coutumes et les traditions. Par exemple, cela est le cas quand un joueur participe aux activités du club, agit en tant que «membre » et accepte les règles spécifiques du groupe et de l'institution (couleurs, statuts, traditions, pratiques, et croyances...). Ici, l'expérience conceptuelle est strictement individuelle et correspond à la construction d'une situation qui est propre au sujet. Étudier le comportement du joueur, c'est étudier son contenu, ses formes, sa cohérence et son histoire. C'est à propos des rapports du comportement et de l'expérience du sujet que Vygotski souligne que la conscience est l'expérience vécue d'expériences vécues (Vygotski, 2003). C'est bien le résultat de la construction de connaissances concrètes acquises par l'usage en contact avec la réalité et prêtes à être mises en pratique.

C'est pourquoi ce concept d'expérience nous semble très important dans la formalisation de l'activité du joueur. Celle-ci comporte des significations, du sens et de l'affectivité qui permettent l'émergence de connaissances. Ces constructions cognitives, émotionnelles et motrices sont révélatrices à la fois dans leur construction et dans leur expression, de la subjectivité du sujet. L'influence de l'expérience du joueur sur l'organisation du jeu et ses actions imprègne toutes ses prises de décisions. Celles-ci s'inscrivent alors dans une recherche d'efficacité qui s'exprime au travers d'une pensée tactique autonome et personnelle (Mouchet, ibid.).

\section{La pensée tactique}

Au début de ce chapitre, nous avons caractérisé stratégie et tactique. Dans ce paragraphe, c'est un autre aspect de la tactique qui sera développé. En effet, la tactique peut être considérée aussi dans une unité dialectique avec la technique. Mais c'est la pensée qui donne un contenu à la tactique des procédés techniques, les transformant en actions. Pendant le match, la pensée, dénommée conventionnellement pensée tactique, a un caractère actif, favorisant l'initiative. Cette forme d'intelligence engagée dans la pratique doit combiner à la fois le flair, la débrouillardise, l'attention vigilante, le sens de l'opportunité,... Dans ce cas, l'accent est toujours mis sur «l'efficacité pratique », en un mot la recherche du succès et de la réussite dans le domaine concret de l'action. Au-delà des apprentissages liés à l'observation du jeu, il est bien évident que cette efficacité pratique doit être souple et déliée dans la mesure où elle est confrontée à des situations constamment changeantes. Elle doit rester ouverte à de nombreux possibles pour s'adapter sans cesse. Pour réussir, le joueur doit concentrer son attention sur le cours du jeu, la configuration en cours telle qu'il s'y trouve engagé, le potentiel de cette situation pour en déceler la cohérence et en profiler son évolution pour prendre des initiatives adéquates ou appropriées.

Il est aussi à noter que tous les sports collectifs restent soumis à des principes empiriques de 
fonctionnement qui se révèlent communs et identiques avec quelques variations propres au volleyball. Ces principes constituent le point de départ, la base. Ils représentent la source de l'action, ils définissent les propriétés invariables sur lesquelles va se greffer la structure fondamentale du déroulement des événements. Norme d'action clairement représentée à l'esprit, le principe guide les joueurs en dirigeant et coordonnant leur activité. "Lois premières, c'est-à-dire essentielles, évidentes et simples, elles sous-tendent, en les régulant, tant en attaque qu'en défense, les rapports des individus au sein de l'équipe et les rapports des deux équipes au cours de l'affrontement. Un principe se pare d'un trait caractéristique : pouvoir être appliqué, acquis et interprété d'une façon identique par les hommes de bon sens »(Bayer, 1979 p. 44). Lorsqu'on analyse la pensée tactique, il faut donc partir de ses formes concrètes spécifiques et des signes qu'elle génère. «Comme l'activité en jeu représente, dans son essence même, la solution de nombreux problèmes apparaissant dans telle ou telle situation, la pensée tactique en tant que processus intellectuel de cette solution est une composante indissociable de cette activité » (Mahlo, 1969 p. 28). Ainsi, toute activité de jeu est un acte forcément tactique, quel que soit le niveau où se situe le joueur, car il consiste à résoudre, pratiquement et dans le respect des règles primaires, un grand nombre de problèmes posés par les diverses situations de jeu. Dans cette complexité, nous pouvons néanmoins distinguer différents niveaux d'abstraction (Mouchet, Amans-Passaga, \& Gréhaigne, 2011) qui consistent à :

- réfléchir collectivement sur les données concrètes de la situation en liaison étroite avec la perception et le jeu ;

- utiliser la pensée tactique liée au jeu mais dépassant la situation concrète. Ceci opère un rapprochement entre la situation telle qu'elle a été reconnue et des règles, des principes, des réponses ;

- recourir à une pensée tactique abstraite qui n'est pas directement liée à l'acte de jeu mais qui s'opère à l'aide de représentations figurées ou de généralisations abstraites pour apporter des réponses aux problèmes posés.

Ces apprentissages s'effectuent soit par imprégnation soit par des exercices appropriés. Du point de vue de la mise en mot dans les échanges entre joueurs, on en reste couramment au niveau des intentions. Pour dépasser ce constat, il faut se poser la question de l'apprentissage de la pensée tactique car une véritable formation à la pensée tactique évite aux formés d'apprendre schématiquement et donc à jouer de même. A ce moment, la capacité à observer, caractériser et à interpréter le plus précisément et objectivement possible ce qui se passe dans une rencontre devient primordiale pour tous.

Le développement d'une pensée tactique

En sport collectif, le développement d'une pensée tactique autonome (Gréhaigne \& Caty, 2014 ; 
Menaut, 1998), permettant d'analyser et d'organiser les réponses face à des problèmes analogues, consiste en l'acquisition et la stabilisation de connaissances, de compétences motrices et d'expériences tactiques nouvelles. Cela vise à la construction d'un système de réponses associées à des configurations prototypiques qui entraine un temps de décision plus rapide entre la perception et le déclenchement d'une réponse adaptée tactiquement. Concrètement, dans ce développement d'une pensée tactique chez le joueur, il nous semble possible de distinguer quatre grandes étapes qu'il convient de concevoir comme pouvant se chevaucher, voire être suivies de sérieux retours en arrière suivant les rapports de forces rencontrés.

- Chez le débutant, la pensée tactique est relativement rudimentaire avec une prise en compte des emplacements des joueurs avec un début de distanciation par rapport à l'action qui peut prendre la forme d'une description différée.

- Dans une autre étape, un joueur débrouillé parvient à décrire les circonstances et les moyens de son action. À partir d'un questionnement du formateur ou d'un débat d'idées (Gréhaigne, 2007), le jeune joueur peut également mettre en rapport des actions en projet ou un début de planification avec le résultat de l'action et ainsi construire un début d'explication des résultats de l'action.

- Dans une troisième étape, les joueurs confirmés peuvent, en analysant leurs actions, décrire les caractéristiques de celles-ci, expliquer les raisons des réussites et voire des échecs ainsi que généraliser les résultats obtenus pour formuler des règles d'action. La pensée tactique, dépassant la situation concrète, opère un rapprochement entre cette configuration telle qu'elle a été reconnue et des généralisations de règles ou de réponses connues.

- Enfin, dans un développement ultérieur, les joueurs experts peuvent se questionner euxmêmes à partir des situations proposées en formulant des hypothèses sur le ou les problèmes à résoudre et sur les moyens à mettre en œuvre. La pensée tactique, qui n'est plus directement liée aux configurations momentanées du jeu, s'opère à l'aide de représentations figurées abstraites.

En constatant les difficultés rencontrées souvent par les joueurs, fréquemment des filles ou des garçons peu expérimentés, il convient de leur proposer un temps d'apprentissage institutionnel d'une durée suffisamment conséquente pour accéder aux connaissances. Chez ces élèves, le développement d'une pensée tactique passe par la possibilité de s'acculturer à des pratiques dont elles ou ils ignorent le sens et ne maîtrisent ni les techniques, ni les tactiques (Poggi, 2016).

Ces étapes constituent une aide pour les formateurs, les joueurs et les élèves dans le nécessaire dialogue pour construire les connaissances indispensables à des échanges explicites. Cela constitue également des repères pour le développement de la pensée tactique et pour la mise en mot que les élèves ou les joueurs vont utiliser lors du débat d'idées. Dans cette mise en mot, le langage ne fait donc pas que décrire le jeu qui lui serait préexistant. C'est lui qui délimite ce que les joueuses et les joueurs peuvent en percevoir et même ce qu'ils ou elles peuvent en penser. 
Pensée tactique et débat entre les joueurs

Le langage est composé d'un signifiant (suite de sons ou de gestes) et d'un signifié ou concept, qui lui donnent sens et autorisent discussions ou débats situés, le plus souvent, avant ou après la séquence de jeu. Vygotsky (1933) développe l'idée qu'il faut être conscient des propriétés de quelques objets pour agir efficacement : c'est la conscience avant. Mais la prise de conscience peut poursuivre son chemin après l'action, dans une sorte de retour réflexif : c'est la conscience après. Dans un autre cadre théorique en psychologie, Caverni (1988) propose les notions de verbalisations préalables, de verbalisations concomitantes et de verbalisations consécutives à une tâche. Les composantes de l'activité mentale identifiables à partir des protocoles verbaux peuvent renvoyer soit à des intentions référées à un but ou à un état futur, soit à des cognitions marquant une attention portée sur des aspects particuliers de la situation présente, soit à des planifications représentant des états explorés mentalement et, enfin, cela peut aboutir à des évaluations exprimant des comparaisons entre différentes possibilités. Pour décider en cours de jeu, Bouthier (2000) parle pour sa part de l'utilisation de trames de variance permettant d'articuler plus finement la pensée stratégique à base de plans et la pensée tactique régulatrice, en conciliant l'organisation collective des actions et l'initiative individuelle au fil des circonstances locales de la situation d'opposition. Ici, l'initiative est au cœur des modes de résolution.

Concernant un joueur expérimenté, l'attitude qu'il nous semble intéressante de rechercher serait le «jouer + réfléchir » dans le double sens d'une réflexion en action (pendant) et d'une réflexion autour du jeu (avant et/ou après). Néanmoins, la pensée tactique est différente selon le moment de ces opérations (Fernandez, 2002 ; Fernandez \& Gréhaigne, 1999). Ainsi :

Pendant le jeu :

- des phases interactives avec plus ou moins de pression et de possibilité de décentration suivant les états psychiques individuels et collectifs pour une réflexion consciente calme ;

- phase arrêtée donnant la possibilité d'échanges brefs mais plus formalisés ;

- phase d'arrêt de jeu en cas d'une blessure ou échanges avec l'entraîneur lors d'une pause.

En dehors du jeu :

- à la mi-temps et/ou entre les rencontres qui redonnent de l'importance aux autres éléments environnementaux de l'équipe et aux types de lieux où les échanges se déroulent.

Toutefois, deux questions importantes restent posées concernant les échanges sur la pensée tactique : Y a-t-il plusieurs cognitions à la clé ? Quelle est la nature des échanges entre les joueurs ? Quels mots pour échanger ? Deleplace (1979) répond à ce problème en proposant, pour l'équipe, la coconstruction d'un référentiel commun de décisions. Cette notion de référentiel commun constitue un 
système cohérent de représentations mentales de la totalité de la logique interne du jeu qui peut être considérée ici comme la trame commune de la pensée tactique des joueurs d'une même équipe et de son entraîneur. Cependant, l'une des principales difficultés à sa mise en œuvre est la diversité des points de vue de tous les acteurs, ce qui nous amène à considérer que l'émergence d'un monde en partie commun aux joueurs est nécessaire. Dans ce cadre, chacun devient en mesure de comprendre la décision prise par un partenaire et donc aussi, dans une certaine mesure, de la prévoir même si tous les joueurs, confrontés au même cas, n'auraient peut-être pas pris ce type de décision.

Apprécier la qualité de la pensée tactique relève d'une évaluation plutôt qualitative, c'est le thème que nous allons examiner dans le paragraphe suivant.

Evaluer la pensée tactique

Quelques questions semblent au cœur d'une évaluation qualitative des résultats produits par les prises de décision stratégiques et tactiques dans une suite de matchs. Avons-nous progressé ? Nos hypothèses originelles sont-elles toujours pertinentes ? Les faits ont-ils changés ? Nos idées ontelles bien fonctionné ? Nous en sommes-nous écartés ? Un bilan commun est certes à effectuer, mais le résultat des rencontres reste un indicateur premier sur la pertinence des décisions en jeu. Fréquemment, les stratégies ne sont pas mises en œuvre selon les aléas des situations de jeu mais, parfois, il faut simplement un moment pour réévaluer l'opposition et se rappeler de l'ensemble des décisions convenues. Pour une évaluation plus quantitative, le nombre de balles jouées ou touchées par l'équipe ou par chaque joueur en fonction de son poste constitue une information importante. Citons également le nombre d'échanges de balle à l'intérieur de l'équipe qui permet d'obtenir un graphe des échanges illustrant les relations privilégiées entre les joueurs et ainsi d'avoir une représentation graphique des circulations habituelles de la balle à l'intérieur de l'équipe dans un type d'opposition donné (Gréhaigne, Billard, \& Laroche, 1999). On peut aussi avoir recours à l'indice de la défensive (rapport Balles Conquises / Balles Perdues) qui met en relation le nombre de balles prises et perdues à l'adversaire. Cet indice varie de la valeur zéro, lorsqu'aucune balle n'est conquise, à une valeur de 1 si le nombre de balles conquises est égal au nombre de balles perdues, et est supérieur à 1 si le nombre de balles conquises est supérieur au nombre de balles perdues. Cet ensemble d'outils fournit des données numériques qui peuvent aider à mieux comprendre comment la pensée tactique a été utilisée.

Néanmoins, la pensée tactique est difficile à apprécier car elle relève, sur le fond, d'une activité humaine sujette aux aléas de l'information, des connaissances, de l'imagination et de la volonté. Actuellement marqué par un certain désintérêt au profit des apprentissages techniques, l'effort doit être maintenu pour donner de véritables compétences tactiques à tous les joueurs. Pour évaluer les différents effets constatés, le recours à la «pensée critique» peut être considéré comme une piste 
très intéressante. «Critical thinking is reflective and reasonable thinking that is focused on deciding what to believe or $d o^{1}{ }^{\gg}$ (Ennis, 1987, p. 10). Ce retour critique doit être compris comme intentionnel et régulateur. Il résulte d'interprétations, d'analyses, d'évaluations partielles voire d'inférences. Des considérations conceptuelles, méthodologiques, critériées ou contextuelles, peuvent servir aussi de base à ce retour critique. Au plan épistémologique, cette pensée critique propose à la fois une dimension cognitive et affective. La dimension cognitive comporte des compétences ou capacités qui sont reliées à̀ l'action et à l'exercice de cette pensée. Pour sa part, la dimension affective repose sur des dispositions ou attitudes critiques en relation avec, si possible, un climat apaisé dans le groupe.

Enfin, une véritable compréhension des interactions entre pensée et pratique renvoie à un double travail. Il s'agit d'identifier les structures objectives utilisées par le joueur, ce qui suppose une mise à distance et, en même temps, de se plonger dans la subjectivité de celui-ci. Dans ce cas de figure, la formation du joueur ambitionne quand même, de découvrir ce qu'il y d'objectif ou de permanent dans cette subjectivité qui permet, au joueur, de répondre aux urgences de la pratique quand il n'a pas le temps d'être rationnel (Gréhaigne, 2009).

L'importance de l'évaluation de la pensée tactique repose sur le fait qu'elle permet de constater des avancées dans les réponses des joueurs et tout en aidant à faire progresser l'organisation du jeu.

\section{Conclusion}

L'objectif de ce chapitre visait à expliciter les notions d'initiative et d'intention avec comme arrière-plan théorique l'importance du développement d'une pensée tactique en relation avec les processus cognitifs conscients. La conscience constitue un des aspects fondamentaux de la genèse du comportement humain. Ainsi, proposer une démarche d'apprentissage visant à développer la compréhension et la généralisation de règles de l'action efficace passe par la conceptualisation et/ou la prise de conscience.

Aussi, quand on recourt à des aspects théoriques conscients, on se place d'un point de vue spéculatif, celui de la pensée génératrice de considérations abstraites qui, le plus souvent, excluent les aléas. Quand on se situe du côté de la pratique, on se place du point de vue de l'action, génératrice de résultats concrets qui, alors, incluent les aléas. Aussi, la théorie est généralement dévalorisée au profit de la pratique dont on vante l'utilité et l'efficacité immédiate. Mais toute théorie n'est pas que spéculation pure. Il y a donc des synergies à établir entre la capacité qu'a le joueur pour agir concrètement sur le jeu et son aptitude à penser le jeu. A partir d'une bonne lecture du rapport de forces et d'une utilisation judicieuse de son expérience, il s'agira d'adopter, pour le joueur, un mode de pen-

\footnotetext{
${ }^{1}$ «La pensée critique est une pensée réfléchie et raisonnable qui se concentre sur décider quoi croire ou faire »
} 
ser attentif aux processus et aux dynamismes en évolution dans les rapports d'opposition afin que ses initiatives soient couronnées de succès. En un mot, prendre des initiatives concrètes en jeu et ne pas en rester aux intentions.

\section{Bibliographie}

Bayer, C. (1979). L'enseignement des jeux sportifs collectifs. Paris: Vigot.

Bourdieu, P. (1980). Le sens pratique. Paris : Les Éditions de Minuit.

Bouthier, D. (2000). La coordination des décisions individuelles; contribution de l'intelligence tactique. Colloque Préparation Olympique L'évolution de la pensée tactique, Noisy le Grand, 14 et 15 novembre 2000.

Bouthier, D., Barthes, D., David, B., \& Gréhaigne, J.F. (1994). Tactical analysis of play combinations in rugby with video-computer : rationalising "French -flair". Paper presented to the Second World Congress of notational analysis. Cardiff, November 1994.

Caverni J.P. (1988). La verbalisation comme source d'observables pour l'étude du fonctionnement cognitif. In J.P. Caverni, C. Bastien, P. Mendelsohn, \& G. Tiberghien (Eds.), Psychologie cognitive, modèles et méthodes (pp. 253-273). Grenoble : P.U.G.

Crozier, M., \& Friedberg, E. (1977). L'acteur et le système. Paris : Seuil.

Deleplace, R. (1979) Rugby de mouvement - Rugby total. Paris : Éducation Physique et Sports.

Fernandez, A. (2002). Rupture et prise d'avantage en sports collectifs, un modèle théorique. eJournal de la Recherche sur l'Intervention en Education Physique et Sportive, 2, 03-17.

Fernandez, A., \& Gréhaigne, J.-F. (1999). Quels référents théoriques pour la modélisation en football ? In J.F. Gréhaigne, N. Mahut, \& D. Marchal (Eds) Qu'apprennent les élèves en faisant des activités physiques et sportives? [CD-ROM]. (Dossier: Apprentissage et activité physique, 4) Besançon : IUFM, Université de Franche-Comté.

Gréhaigne, J.-F. (Ed.). (2007). Configurations du jeu, débat d'idées et apprentissage des sports collectifs. Besançon : Presses de 1'Université de Franche-Comté.

Gréhaigne, J.-F. (Ed.) (2014). L’intelligence tactique : quelques données. In J.-F. Gréhaigne (Ed.) L’intelligence tactique. Des perceptions aux décisions tactiques en sports collectifs (pp. 25-40). Besançon : Presses de l'Université de Franche-Comté.

Gréhaigne, J.-F., \& Caty, D. (2014). Développer la pensée tactique à l'école. In J.-F. Gréhaigne (Ed.) L'intelligence tactique. Des perceptions aux décisions tactiques en sports collectifs (pp. 279-300). Besançon : Presses de l’Université de Franche-Comté. 
Gréhaigne, J.-F., \& Dietsch, G. (2015). Quelques aspects théoriques de la didactique des sports collectifs. Préparation aux concours de recrutement. eJRIEPS, Hors Série $N^{\circ} 1.128$ pages.

Gréhaigne, J.-F., \& Godbout, P. (1995). Tactical knowledge in team sports from a constructivist and cognitivist perspective. Quest, 47, 490-505.

Gréhaigne, J.-F., \& Godbout, P. (2014). Dynamic systems theory and team sport coaching Quest, 66 (1), 96-116.

Gréhaigne, J.-F. , Billard, M., \& Laroche, J.Y. (1999). L'enseignement des jeux sportifs collectifs à l'école. Conception, construction, évaluation. Bruxelles : De Boeck. (Collection Sciences et Pratiques du Sport).

Gréhaigne, J.-F., Godbout, P., \& Bouthier, D. (1999). The foundations of tactics and strategy in team sports. Journal of Teaching in Physical Education, 18, 159-174.

Ennis, R. H. (1987). A taxonomy of critical thinking dispositions and abilities. In J. Baron \& R. Sternberg (Eds.) Teaching thinking skills: Theory and practice ( pp. 9-26). NY : W.H. Freeman,.

Le Moigne, J.-L. (1999). La modélisation des systèmes complexes. Paris : Dunod.

Mahlo, F. (1969). Acte tactique en jeu. Paris, France: Vigot.

Menaut, A. (1998). Le réel et le possible dans la pensée tactique. Talence : Presses Universitaires de Bordeaux.

Mouchet, A. (2003). Caractérisation de la subjectivité dans les décisions tactiques des joueurs d'élite 1 en rugby. Thèse de doctorat en STAPS (non publiée). Université Bordeaux 2.

Mouchet, A., Amans-Passaga, C., \& Gréhaigne, J.-F. (2011). L'approche technologique. In M. Musard, M. Loquet \& G. Carlier (Éds.), Sciences de l'intervention en EPS et en sport. Résultats de recherches et fondements théoriques (pp. 201-222). Paris : Éditions EP\&S.

Poggi, M.-P. (2016). Handball scolaire pour les filles en EPS. Quels risques d'inégalités ? In J.-F. Gréhaigne (Ed.), Les objets de la technique. De la compétence motrice à la tactique individuelle (pp. 250-266). Besançon : PUFC.

Vygotski, L. (2003). Conscience, inconscient, émotions. Paris : La Dispute.

Vygotski, L. (1933). Pensée et langage (traduction de Françoise Sève et avant-propos de Lucien Sève), suivi de «Commentaires sur les remarques critiques de Vygotski » de Jean Piaget, (Collection «Terrains», Éditions Sociales, Paris, 1985) ; Réédition, Paris : La Dispute, 1997. 